\title{
Volumetric analysis of syringomyelia following hindbrain decompression for Chiari malformation Type I: syringomyelia resolution follows exponential kinetics
}

\author{
Jean-Valery Coumans, M.D., Brian P. Walcott, M.D., William E. Butler, M.D., \\ Brian V. Nahed, M.D., and Kristopher T. Kahle, M.D., Ph.D. \\ Department of Neurosurgery, Massachusetts General Hospital and Harvard Medical School, Boston, \\ Massachusetts
}

Object. Resolution of syringomyelia is common following hindbrain decompression for Chiari malformation, yet little is known about the kinetics governing this process. The authors sought to establish the volumetric rate of syringomyelia resolution.

Methods. A retrospective cohort of patients undergoing hindbrain decompression for a Chiari malformation Type I with preoperative cervical or thoracic syringomyelia was identified. Patients were included in the study if they had at least 3 neuroimaging studies that detailed the entirety of their preoperative syringomyelia over a minimum of 6 months postoperatively. The authors reconstructed the MR images in 3 dimensions and calculated the volume of the syringomyelia. They plotted the syringomyelia volume over time and constructed regression models using the method of least squares. The Akaike information criterion and Bayesian information criterion were used to calculate the relative goodness of fit. The coefficients of determination $R^{2}$ (unadjusted and adjusted) were calculated to describe the proportion of variability in each individual data set accounted for by the statistical model.

Results. Two patients were identified as meeting inclusion criteria. Plots of the least-squares best fit were identified as $4.01459 e^{-0.0180804 x}$ and $13.2556 e^{-0.00615859 x}$. Decay of the syringomyelia followed an exponential model in both patients $\left(\mathrm{R}^{2}=0.989582\right.$ and 0.948864$)$.

Conclusions. Three-dimensional analysis of syringomyelia resolution over time enables the kinetics to be estimated. This technique is yet to be validated in a large cohort. Because syringomyelia is the final common pathway for a number of different pathological processes, it is possible that this exponential only applies to syringomyelia related to treatment of Chiari malformation Type I. (DOI: 10.3171/2011 .6.FOCUS11106)

$\begin{array}{lccccc}\text { KEY WoRdS } & \bullet & \begin{array}{c}\text { Chiari malformation } \\ \text { MR imaging }\end{array} & \bullet & \text { volumetrics }\end{array}$

Coriter HIARI malformation Type I is defined as caudal displacement of the cerebellar tonsils below the level of the foramen magnum, causing compression of the brainstem and upper cervical cord. The diagnostic criteria have been debated because the absolute degree of descent below the level of the foramen magnum may be less important than the degree of compression of the cervicomedullary junction. Individuals can remain asymptomatic despite a significant descent of the tonsils, and conversely, patients can be symptomatic with little to no caudal displacement of the tonsils. ${ }^{10}$

Compression of the spinal cord, whether caused by CM-I, by a disc herniation, or by a mass lesion, has been associated with syringomyelia. The mechanism of syringomyelia formation is poorly understood, and several hypotheses have been proposed. , $8,9,11$ Some have postulated

Abbreviation used in this paper: $\mathrm{CM}-\mathrm{I}=$ Chiari malformation Type I. that the drainage of CSF produced by the ependymal cells of the central canal is blocked, leading to a caudal accumulation of fluid. Others have argued that, in the case of CM-I, occlusion of the foramen magnum causes the intracranial CSF pulsations to be driven into the central canal or into the spinal Virchow-Robin spaces instead of its normal egress via the spinal subarachnoid space. ${ }^{8} \mathrm{Al}$ though the theory postulated by Oldfield et al. may be the most accurate, ${ }^{7,14}$ the pathogenesis may be an amalgamation of many theories, including those by Gardner, ${ }^{5}$ Williams, ${ }^{22}$ Ball and Dayan, ${ }^{4}$ Aboulker, ${ }^{1,2}$ and Greitz. ${ }^{6}$

Regardless of mechanism, it has long been recognized that restoration of CSF flow around the spinal cord is associated with a resolution of syringomyelia. ${ }^{21}$ The kinetics governing resolution of syringomyelia following decompression of the spinal cord remains to be elucidated. In this study, we performed a volumetric analysis of syringomyelia after CM-I decompression and examined the pattern of decay. 


\section{Methods}

We identified patients with CM-I and syringomyelia who underwent hindbrain decompression. Patients were included for analysis if there were at least 3 postoperative MR images detailing the entire extent of their preoperative syringomyelia, allowing a curve to be plotted. The volume at each time point was calculated by importing DICOM data into OsiriX open-source software (Pixmeo) and identifying the syringomyelia as the region of interest (Fig. 1). Axial coherent oscillatory state acquisition for the manipulation of image contrast (COSMIC) sequences were used for this, allowing for optimal differentiation between the CSF and spinal cord. ${ }^{20}$ The $3 \mathrm{D}$ volume was rendered and calculated (Fig. 2). ${ }^{16}$ We then plotted the syringomyelia volume over time and constructed regression models using the method of least squares. ${ }^{12}$ The Akaike information criterion ${ }^{3}$ and Bayesian information criterion ${ }^{17}$ were used to calculate the relative goodness of fit. The coefficients of determination $R^{2}$ (unadjusted and adjusted) were calculated to describe the proportion of variability for each individual data set accounted for by the statistical model. An independent biostatistician validated the study methodology and data analysis.

\section{Results}

Two patients were identified as meeting inclusion criteria. Plots of the least-squares best fit were identified as $4.01459 e^{-0.0180804 x}$ for the patient in Case 1 and 13.2556 $e^{-0.00615859 x}$ for the patient in Case 2 (Fig. 3). Decay of the syringomyelia followed an exponential model closely in both patients as evidenced by Akaike information criterion, Bayesian information criterion, $R^{2}$, and $R^{2}$-adjusted in Case 1 of 4.77068, 2.92957, 0.989582, and 0.979164, respectively. Case 2 had comparable values of 18.4951 , $16.654,0.948864$, and 0.897728 , respectively.

\section{Discussion}

It is unclear why syringomyelia only develops in a subset of individuals with CM-I. The condition may be related to the degree of compression rather than to the absolute

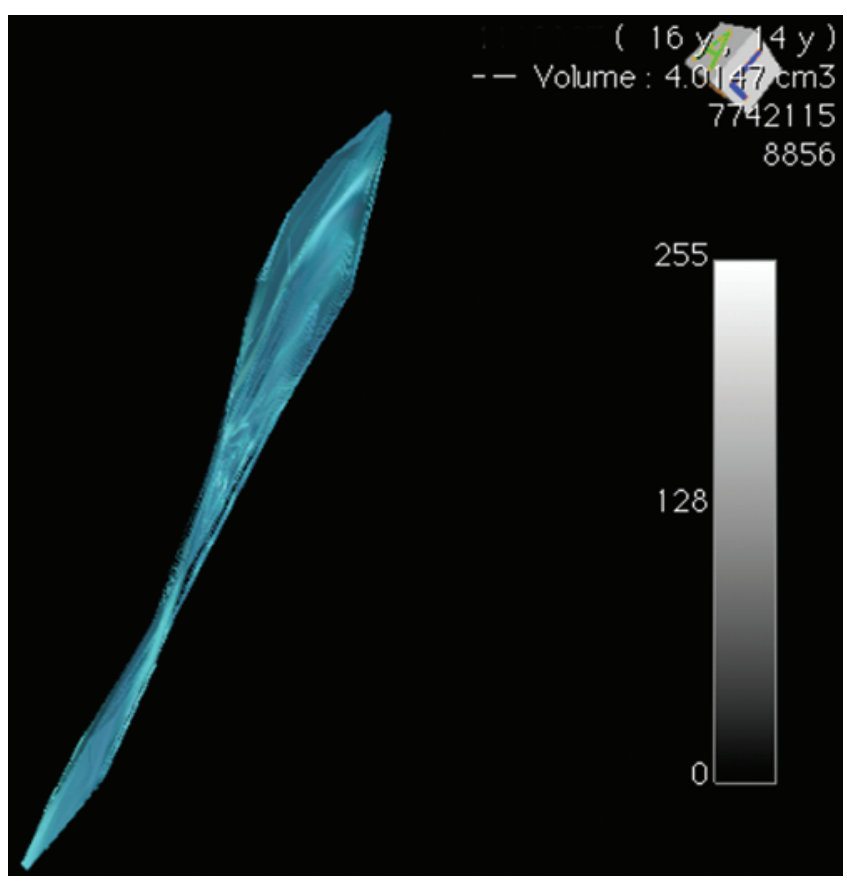

FIG. 2. Three-dimensional reconstruction. Using consecutive regions of interest, a 3D volume was rendered and quantitatively calculated.

caudal migration of the cerebellar tonsils or to unidentified factors. Other compressive lesions at the cervicomedullary junction can cause syringomyelia. ${ }^{19}$ The resolution of syringomyelia after CM-I decompression, however, is well characterized and occurs in the majority of cases..$^{21}$ Instances in which syringomyelia fails to resolve postoperatively can be due to arachnoid webbing, extra- or intradural adhesions or scarring, or failure to adequately decompress the craniocervical junction. ${ }^{7,18}$ Interestingly, syringomyelia can also resolve spontaneously on rare occasions. ${ }^{9}$ Wetjen and coauthors ${ }^{21}$ have estimated the rate of syringomyelia resolution by examining the postoperative images in patients after CM-I decompression. Based on the largest anteroposterior diameter of the syringomyelia, the authors concluded that the median time to greater than $50 \%$ narrowing of the syringomyelia was 3.6 months postoperatively, whereas the

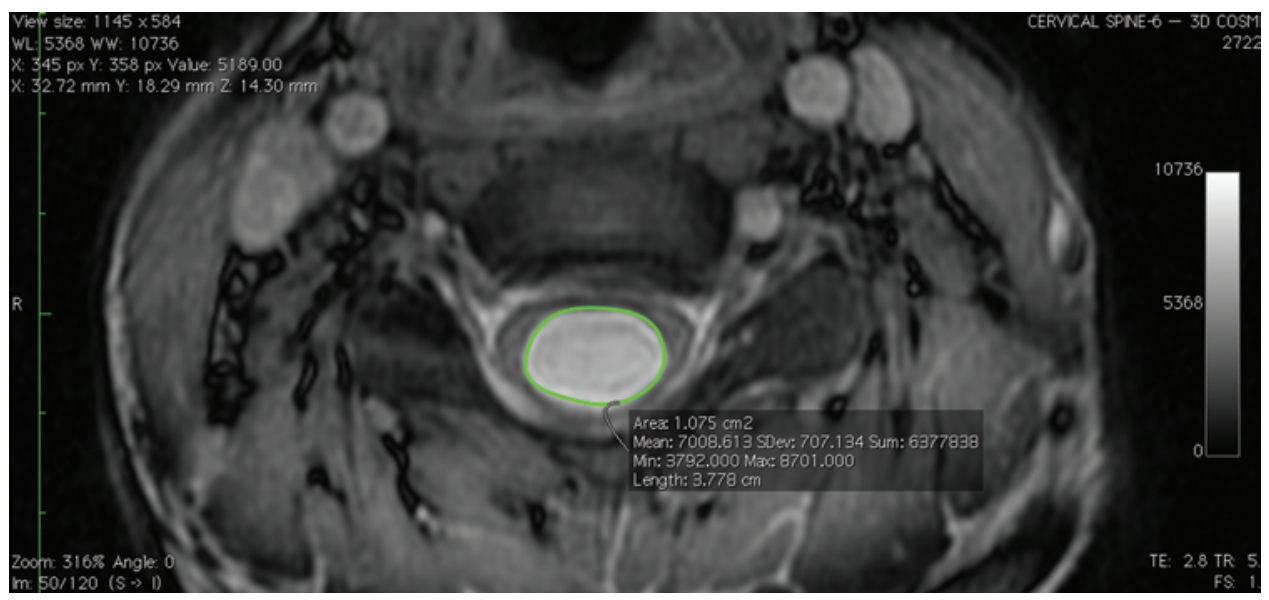

FIG. 1. Region of interest selection. Using OsiriX software, we identified the syringomyelia as the region of interest. 


\section{Volumetric analysis of syringomyelia}

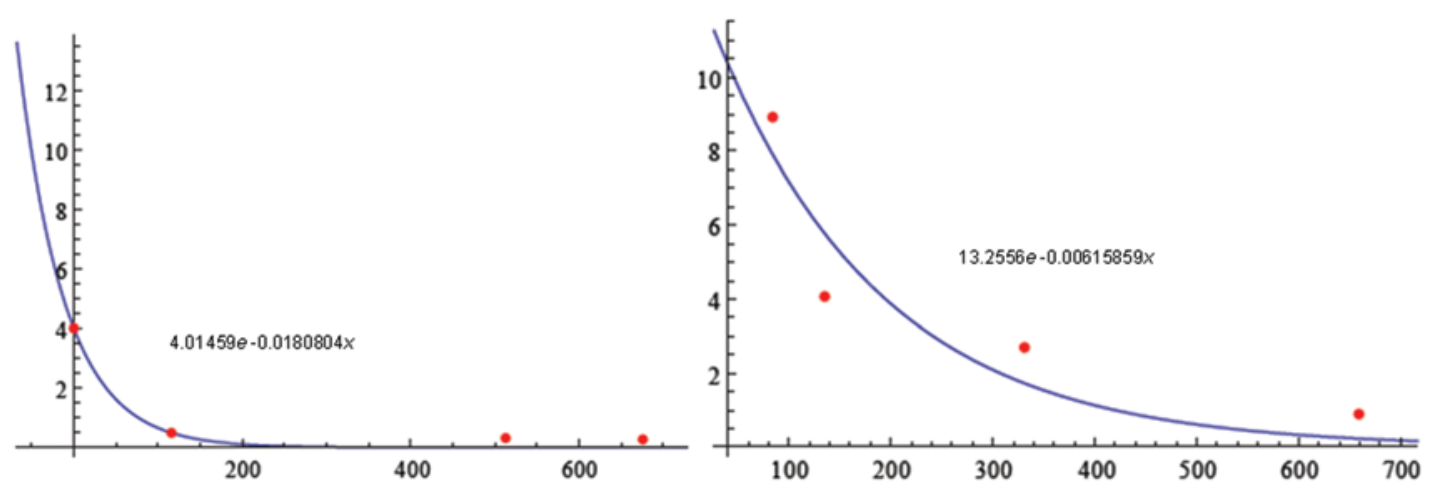

FIG. 3. Rate of syringomyelia decay. Plots of the least-squares best fit in Cases 1 (left) and 2 (right). $x$ axis = number of days following surgery; y axis = syringomyelia volume $\left(\mathrm{cm}^{3}\right)$. Left: Wolfram Alpha LLC. 2011. Wolfram|Alpha. http://www. wolframalpha.com/input $/$ i $=$ exponential $+f i t+\{0 \% 2 C+4.0147\} \% 2 C+\{116 \% 2 C+0.4918\} \% 2 C+\{513 \% 2 C 0.3165\} \% 2 C+$ \{676\%2C0.2689\} (Accessed July 14, 2011). Right: Wolfram Alpha LLC. 2011. Wolfram|Alpha. http://www.wolframalpha.com/ input/?i=exponential+fit $+\{84 \% 2 \mathrm{C} 8.916\} \% 2 \mathrm{C}+\{135 \% 2 \mathrm{C}+4.0665\} \% 2 \mathrm{C}+\{331 \% 2 \mathrm{C} 2.6985\} \% 2 \mathrm{C}+\{659 \% 2 \mathrm{C}+0.8934\}$ (Accessed July 14, 2011).

mean time was 6.5 months. To our knowledge, these are the best data available on the rate of syringomyelia resolution after CM-I decompression, although volumetric analysis related to Chiari malformation and interval changes over time go back as far as Nyland and Krogness. ${ }^{13}$

Most authors describe a syringomyelia by its width or by its cranial to caudal extent. Although measuring its diameter on axial imaging or its longitudinal extent over time allows one to estimate the size of a syringomyelia, it is not a direct measurement of volume. Because a syrinx is an irregular 3D structure, MR imaging-based volume measurement as suggested in this study may be a more precise measurement than linear dimension estimates. In this study, we plotted the volume of a syringomyelia over time and found that its resolution follows exponential decay. Potential implications of this technique are that, if validated, volumetric resolution of a syrinx may be correlated with the adequacy of hindbrain decompression.

The factors governing the rate of syringomyelia resolution may be complex and depend on the viscosity of the syringomyelia fluid, the shape of the cavity, the elasticity of the spinal cord, and the transmural pressure. Although flow in elastic tubes such as blood vessels has been modeled, ${ }^{15}$ little is known about the kinetics of syringomyelia. It is likely that the kinetics are more complex than the simple emptying of a container or balloon (Fig. 3). Syringomyelia can take months to empty after surgery, suggesting that complex factors affect this process. Ultimately, the volume of a syringomyelia represents the net difference between factors governing its accumulation and those affecting its drainage, and volumetric measurements over time as in the current study do not allow one to characterize these opposing forces. It appears that emptying of the syringomyelia is not limited by the outflow rostrally through the central canal. If this were the case (simply losing fluid volume through a very narrow central canal), one would expect the bottleneck at the rostral aspect to be the limiting factor and one would see a linear decline in volume over time. In this instance, the exponential decay suggests that the rate of loss is dependent on the residual volume: the greater the residual volume, the greater the rate of egress.

\section{Conclusions}

Three-dimensional analysis of syringomyelia resolution following surgery over time enables the kinetics to be estimated and can be described as exponential decay. This analysis should be interpreted as an unvalidated metric that will need to be assessed in a larger group to establish a generalizable mechanism of syrinx resolution. Because syringomyelia is the final common pathway for a number of different pathological processes, it is possible that this exponential decay only applies to syringomyelia related to treatment of CM-I.

\section{Disclosure}

This work was conducted with support from Harvard Catalyst I The Harvard Clinical and Translational Science Center (National Institutes of Health Award No. UL1 RR 025758 and financial contributions from Harvard University and its affiliated academic health care centers). The content is solely the responsibility of the authors and does not necessarily represent the official views of Harvard Catalyst, Harvard University and its affiliated academic health care centers, the National Center for Research Resources, or the National Institutes of Health. The authors used the open-source software OsiriX Imaging Software for volumetric analysis. This open-source version does not have FDA 510(k) clearance for clinical use in the US.

Author contributions to the study and manuscript preparation include the following. Conception and design: Walcott, Coumans. Acquisition of data: Walcott, Coumans. Analysis and interpretation of data: Walcott, Coumans, Butler. Drafting the article: all authors. Critically revising the article: all authors. Reviewed submitted version of manuscript: all authors. Approved the final version of the manuscript on behalf of all authors: Walcott. Statistical analysis: Walcott, Coumans. Study supervision: Coumans.

\section{References}

1. Aboulker J: [Syringomyelia and intra-rachidian fluids. VIII. Normal fluids movements and pathology out rachis.] Neurochirurgie 25 (Suppl 1):73-80, 1979 (Fr)

2. Aboulker J: [Syringomyelia and intra-rachidian fluids. X. Rachidian fluid stasis.] Neurochirurgie 25 (Suppl 1):98-107, 1979 (Fr)

3. Akaike H: A new look at the statistical model identification. IEEE Transactions on Automatic Control 19:716-723, 1974 


\section{J. V. Coumans et al.}

4. Ball MJ, Dayan AD: Pathogenesis of syringomyelia. Lancet 2:799-801, 1972

5. Gardner WJ: Hydrodynamic mechanism of syringomyelia: its relationship to myelocele. J Neurol Neurosurg Psychiatry 28:247-259, 1965

6. Greitz D: Unraveling the riddle of syringomyelia. Neurosurg Rev 29:251-264, 2006

7. Heiss JD, Suffredini G, Smith R, DeVroom HL, Patronas NJ, Butman JA, et al: Pathophysiology of persistent syringomyelia after decompressive craniocervical surgery. Clinical article. J Neurosurg Spine 13:729-742, 2010

8. Koyanagi I, Houkin K: Pathogenesis of syringomyelia associated with Chiari type 1 malformation: review of evidences and proposal of a new hypothesis. Neurosurg Rev 33:271285,2010

9. Kyoshima K, Bogdanov EI: Spontaneous resolution of syringomyelia: report of two cases and review of the literature. Neurosurgery 53:762-769, 2003

10. Lee JH, Chung CK, Kim HJ: Decompression of the spinal subarachnoid space as a solution for syringomyelia without Chiari malformation. Spinal Cord 40:501-506, 2002

11. Levy EI, Heiss JD, Kent MS, Riedel CJ, Oldfield EH: Spinal cord swelling preceding syrinx development. Case report. J Neurosurg 92 (1 Suppl):93-97, 2000

12. Marquardt DW: An algorithm for least-squares estimation of nonlinear parameters. J Soc Ind Appl Math 11:431-441, 1963

13. Nyland H, Krogness KG: Size of posterior fossa in Chiari type 1 malformation in adults. Acta Neurochir (Wien) 40:233242, 1978

14. Oldfield EH, Muraszko K, Shawker TH, Patronas NJ: Pathophysiology of syringomyelia associated with Chiari I malfor- mation of the cerebellar tonsils. Implications for diagnosis and treatment. J Neurosurg 80:3-15, 1994

15. Rosar ME, Peskin CS: Fluid flow in collapsible elastic tubes: a three-dimensional numerical model. New York J Math 7: 281-302, 2001

16. Rosset A, Spadola L, Ratib O: OsiriX: an open-source software for navigating in multidimensional DICOM images. J Digit Imaging 17:205-216, 2004

17. Schwarz G: Estimating the dimension of a model. Ann Stat 6: 461-464, 1978

18. Souweidane MM: Editorial. Chiari malformation Type I. J Neurosurg Spine 13:727-728, 2010

19. Suyama K, Ujifuku K, Hirao T, Takahata H, Ito M, Yonekura $\mathrm{M}$, et al: Symptomatic syringomyelia associated with a dermoid tumor in the posterior fossa. Neurol Med Chir (Tokyo) 49:434-437, 2009

20. Vertinsky AT, Krasnokutsky MV, Augustin M, Bammer R: Cutting-edge imaging of the spine. Neuroimaging Clin $\mathbf{N}$ Am 17:117-136, 2007

21. Wetjen NM, Heiss JD, Oldfield EH: Time course of syringomyelia resolution following decompression of Chiari malformation Type I. J Neurosurg Pediatr 1:118-123, 2008

22. Williams B: The distending force in the production of communicating syringomyelia. Lancet 2:41-42, 1970

Manuscript submitted May 15, 2011.

Accepted June 23, 2011.

Address correspondence to: Brian P. Walcott, M.D., Massachusetts General Hospital, 55 Fruit Street, White Building, Room 502, Boston, Massachusetts 02114. email: walcott.brian@mgh.harvard. edu. 